\title{
Left main dissection provoked by guiding catheter during primary percutaneous coronary intervention
}

\author{
Tomislav Jakljević*, Vjekoslav Tomulić \\ University Hospital Center Rijeka, Rijeka, Croatia
}

Left main dissection provoked by guiding catheter (iatrogenic) is rare, possible and potentially fatal complication during diagnostic coronary angiography and percutaneous coronary intervention. The incidence in literature is around $0.1 \%$, although there is an objective chance that this complication remains underreported ${ }^{1}$. Left main dissection could be consequence of inadvertent catheter handling, but sometimes just a result of diffuse atherosclerosis and "fragile" coronary arteries ${ }^{2}$. It can be treated conservatively, with stent implantation or coronary artery bypass grafting ${ }^{3}$. We report a case of iatrogenic left main dissection in primary percutaneous coronary intervention, which, to our knowledge, have never been reported.

A 70-years old female patient was transferred to our hospital because of anteroseptolateral STEMI. Unfortunately "pain to needle" time was over 10 hours because of technical transportation problems. Her comorbidities were diabetes mellitus and arterial hypertension. On admission to our Emergency department she was in Killip 1 stage, blood pressure was $140 / 90 \mathrm{~m} \mathrm{mHg}$. Urgent angiogram was performed and showed acute occlusion of proximal LAD. EBU 3.5 guiding catheter was placed and the lesion successfully crossed with a "workhorse" coronary wire. After balloon predilatation successful stenting of the lesion was performed. Final control angiogram presented catheter induced dissection of left main towards strong circumflex branch. We placed another "workhorse" coronary wire in true circumflex lumen. Direct stenting of left main and proximal circumflex artery was performed with TIMI 3 flow. After recrossing the LAD ostium we performed plain balloon dilatation and achieved an optimal result. On the third hospitalization day the patient developed cardiogenic shock with total atrioventricular block. Inoconstrictor support was started and percutaneous temporary pacemaker implanted. Control coronary angiogram showed patent left coronary artery and we decided to implant intraaortic balloon pump. At that moment pulseless electrical activity occurred. Resuscitation measures according to European resuscitation council protocol were performed but unfortunately without any success.

KEYWORDS: iatrogenic dissection, left main stem, percutaneous coronary intervention.

\section{Received: $10^{\text {th }}$ Mar 2013}

*Address for correspondence: Klinički bolnički centar Rijeka, Tome Stržića 3, HR51000 Rijeka, Croatia.

Phone: $+385-51-407-149$

Fax: $+385-51-218-059$

E-mail: tjakljev@inet.hr

\section{Literature}

1. Boyle AJ, Chan M, Dib J, Resar J. Catheter-induced coronary artery dissection: risk factors, prevention and management. J Invasive Cardiol. 2006;18(10):500-3.

2. Eshtehardi $\mathrm{P}$, Adorjan $\mathrm{P}$, Togni $\mathrm{M}$, et al. latrogenic left main coronary artery dissection: incidence, classification, management, and long-term follow-up. Am Heart J. 2010;159(6):1147-53.

3. Cheng Cl, Wu CJ, Hsieh YK, et al. Coronary intervention for iatrogenic left main coronary artery dissection. Int J Cardiol. 2008; 126(2):177-82.
} 